\title{
Using Spatial Data Mining to Predict the Solvability Space of Preconditioned Sparse Linear Systems
}

\author{
Shuting Xu, SangBae Kim and Jun Zhang \\ 1. School of Science and Technology, Georgia Gwinnett College, Lawrenceville, Georgia 30043, USA \\ 2. Department of Mathematics, Hannam University, Daejeon 306791, South Korea \\ 3. Department of Computer Science, University of Kentucky, Lexington, Kentucky 40506, USA
}

\begin{abstract}
The solution of large sparse linear systems is one of the most important problems in large scale scientific computing. Among the many methods developed, the preconditioned Krylov subspace methods [1] are considered the preferred methods. Selecting an effective preconditioner with appropriate parameters for a specific sparse linear system presents a challenging task for many application scientists and engineers who have little knowledge of preconditioned iterative methods. The purpose of this paper is to predict the parameter solvability space of the preconditioners with two or more parameters. The parameter solvability space is usually irregular, however, in many situations it shows spatial locality, i.e. the parameter locations that are closer in parameter space are more likely to have similar solvability. We propose three spatial data mining methods to predict the solvability of ILUT which make usage of spatial locality in different ways. The three methods are MSC (multi-points SVM classifier), OSC (overall SVM classifier), and OSAC (overall spatial autoregressive classifier). The experimental results show that both MSC and OSAC can obtain 90\% accuracy in prediction, but OSAC is much simpler to implement. We focus our work on ILUT preconditioner [2], but the proposed strategies should be applicable to other preconditioners with two or more parameters.
\end{abstract}

Key words: Preconditioner, prediction, solvability, SVM, spatial autoregressive model.

\section{Introduction}

There are many scientific applications that need to solve large sparse linear systems with sparse coefficient matrices. The class of preconditioned Krylov subspace methods [1] (with a Krylov iterative solver and a preconditioner) is considered the preferred methods in this field. The preconditioners employed in the preconditioned iterative solvers usually determine the overall convergence rate of the iteration procedure $[4,16]$. However, choosing a good preconditioner for a specific sparse matrix arising from a particular application is the combination of art and science, and presents a formidable challenge for many design engineers and application scientists who have little knowledge of preconditioned iterative methods.

Corresponding author: Shuting $\mathrm{Xu}, \mathrm{Ph} . \mathrm{D}$., associate professor, research fields: machine learning, information security, and scientific computing.
Current approaches to recommending iterative solvers and preconditioners are quantitative and categorical. Although mathematical theorems can be proved to guarantee the convergence of certain preconditioned iterative methods for a given model problem, they are not very useful for solving problems encountered in practical applications. Thus a recommendation approach that permits tradeoffs and that can be built and modified incrementally, based on increased knowledge, is certainly helpful. The success of the software environment approach for other problems in scientific computing [13] suggests that it can be very useful when a recommendation system or a software environment approach is applied to the problem of finding the right path to solving a sparse linear system.

The first idea of using matrix features and data mining techniques to predict the possibility of solving a sparse linear system was proposed in Ref. [5]. We 
are now working on an OPPS (online preconditioner prediction system), which can predict whether a given sparse matrix can be solved by a certain preconditioned solver. The main structure of the OPPS is described in Fig. 1. After a matrix is submitted, its features are computed and saved in a feature matrix. Some data mining algorithms are applied on the feature matrix to find out models, rules or other kinds of knowledge about the matrix features and their relationship with the preconditioned solvers. Such models, rules or knowledge are used to predict the solving status of the matrix, such as whether the sparse matrix can be successfully solved by a particularly preconditioned solver, if not, which kind of problems will occur, etc. The procedures like mining the feature matrix are done in background and are repeated after some new matrices are submitted to the system. With more and more matrices added to the system, the prediction accuracy will improve.

We have made significant progress in using data mining techniques and matrix features to predict the solving status of sparse matrices by matrix structure-based incomplete LU type preconditioners such as ILU(0) and ILU(k) [6]. The preconditioner we work on in this paper is ILUT [2], one of the popular preconditioners with many successful applications. Unlike ILU(0) or ILU(k), ILUT needs two preset parameters and it only works well under some sets of values of these parameters. The parameter solvability space is usually irregular, however, in many situations

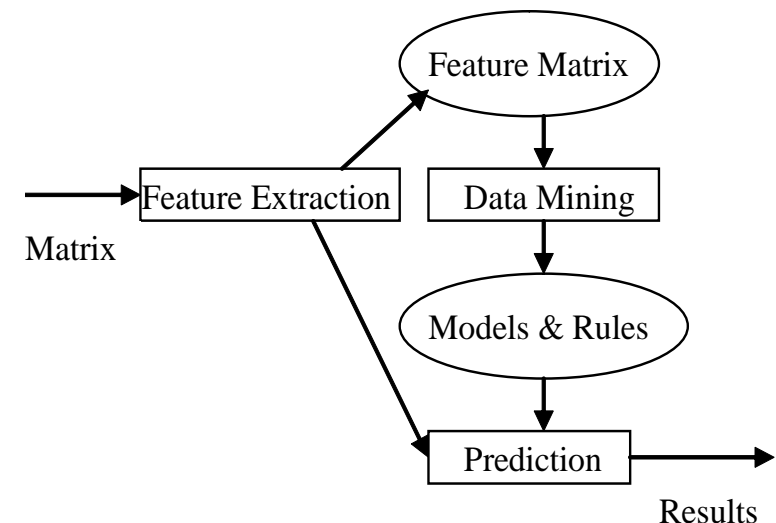

Fig. 1 Structure of OPPS. it shows spatial locality, i.e. parameter locations that are closer in parameter space are more likely to have similar solvability. In this paper, we propose three methods to predict the solvability of ILUT which make usage of spatial locality in different ways. The three methods are MSC (multi-points SVM classifier), OSC (overall SVM classifier), and OSAC (overall spatial autoregressive classifier). We have conducted the experiments to test and compare the prediction accuracy of these three methods.

The rest of the paper is organized as follows. We introduce the matrix features extracted for solvability prediction and the feature selection method applied in Section 2. In Section 3, we describe in detail the three methods for predicting ILUT parameter space of solution. The computational experiments are carried out and the results are discussed in Section 4. We sum up this paper in Section 5.

\section{Matrix Feature Extraction and Selection}

\subsection{Feature Extraction}

The matrix features used are directly related to the precision of the prediction system. We compute the features of a matrix first and then use such features to predict the solving status of the matrix. The features of a matrix are a reflection of its sparsity, locations and the size of the nonzero elements. We extract about 66 features related to matrix structure, value, bandwidth and diagonal statistics. There may be other useful features that we can extract in the future and add them to the feature space.

\subsubsection{Structure}

This group of features describes the distribution of nonzero entries of a matrix. For example, we compute the sparsity rate (the number of nonzero elements divided by the number of all elements) of the whole matrix (nnzrt), of the lower diagonal part (lowfillrt), of the upper diagonal part (upfillrt) and of the main diagonal (diagfillrt). Other features include the average nonzero entries per row (avnnzprow) and the standard deviation (sdavnnzprow), the average 
nonzero entries per column (avnnzpcol) and the standard deviation (sdavnnzpcol), the maximum and minimum number of nonzero elements per column and per row (maxnnzpcol, minnnzpcol, maxnnzprow, minnnzprow). We also calculate the total number of non-void diagonals (nzdiags), i.e., the number of diagonals which has at least one nonzero element among the $2 n-1$ diagonals of the matrix, where $n$ is the dimension of the matrix.

The attribute symmc measures whether a matrix is symmetric, i.e., $A=A^{T}$. relsymm describes the relative symmetry rate of a matrix. It is the ratio of the number of elements that match divided by $n n z$, which is the number of nonzero entries of the matrix. An element $a(i, j)$ in the matrix $A$ matches if it satisfies the following condition: if $a(i, j)$ is nonzero then $a(j, i)$ is nonzero. If a matrix is a normal matrix, normal is equal to 1 , otherwise it is 0 . The attribute blocksize reflects whether a matrix has a block structure or not. The matrix has a block structure if it consists of square blocks that are dense. The value of blocksize greater than one represents the size of the largest block.

\subsubsection{Value}

The attributes in this group sum up the value distribution of a matrix, e.g., one norm (onenorm), infinity norm (infnorm), and Frobenius norm (frnorm) of a matrix. This group also includes the minimum of the sum of the columns (minonenorm), the minimum of the sum of the rows (mininfnorm), the Frobenius norm of the symmetric part of a matrix (symfnorm), and of the unsymmetric part (nsymfnorm).

We also consider the average value of all nonzero entries (avnnzval) and the standard deviation (sdavnnzval), the average of the main diagonal entries (avdiag) and the standard deviation (sdavdiag), the average of the upper triangular entries (avuptrig) and the standard deviation (sdavuptrig), as well as the average of the lower triangular entries (avlowtrig) and the standard deviation (sdavlowtrig).

\subsubsection{Bandwidth}

This group of features describes the bandwidth of a matrix. Bandwidth provides a measure of the clustering of nonzero entries about the main diagonal. Lower bandwidth of a matrix (lowband) is defined as the largest value of $i-j$, where $a(i, j)$ is nonzero. Similarly, upper bandwidth of a matrix (upband) is defined as the largest value of $j-i$. Maximum bandwidth (maxband) is defined as $\max (\max (j)-\min (j))$. Average bandwidth (avband) is defined as the average width of all columns.

\subsubsection{Diagonal}

The features in this group are related with the diagonal. For instance, they include the average distance from each entry to the diagonal (avdisfd) and the standard deviation (sdavdisfd), the average of the difference from each of the entry to its diagonal value (avvalfd) and the standard deviation (sdavvalfd), the average of the difference from the largest value in a row to the diagonal value (avmaxvalfd) and the standard deviation (sdavmaxvalfd).

Other features in this category include the percentage of weakly diagonally dominant columns (diagdomcol) and the percentage of weakly diagonally dominant rows (diagdomrow). diagvalrate is the ratio of the minimum absolute diagonal element value (except zero) to the maximum absolute diagonal element value.

\subsubsection{Others}

We also include some other features, such as strzpiv - the number of structural zero pivots, (a structural zero pivot is a null column above or null row to the left of a zero diagonal element); zpivrow - whether a matrix has a null row to the left of a zero diagonal element; zpivcol — whether a matrix has a null column above a zero diagonal element; szvdiag - the smallest nonzero diagonal element with the dot product of its left vector and up vector being zero; minvalcol - the minimum of the smallest nonzero value in each column with a zero diagonal element. 
For more detailed description on the identified matrix features, please see Ref. [6].

\subsection{Feature Selection}

Some of the matrix features extracted in the previous section are related and even redundant. Applying feature selection may reduce the dataset dimension, speedup computation, remove noise, and improve prediction accuracy. PCA (principal components analysis) [3] is a widely used method to reduce the dimension of datasets, and we use it here for feature selection. The steps of applying PCA are as follows:

(1) For a given zero mean $m \times n$ matrix $A$ ( $m$ attributes and $n$ records), calculate its covariance matrix cov $=\frac{1}{n-1} A A^{T}$;

(2) Find the eigenvectors $P C$ and eigenvalues $V$ of cov;

(3) Order the eigenvalues $V$ in decreasing order and sort the eigenvectors $P C$ accordingly;

(4) Pick the $k$ first eigenvectors;

(5) Project to the original data.

\section{ILUT Parameter Space Prediction}

ILUT is an incomplete LU preconditioner with double dropping strategies. It is one of the popular preconditioners with many successful applications. PGMRES with ILUT can solve some sparse linear systems that would fail with other preconditioners like ILU0 (e.g., matrix F2DB). With ILUT, PGMRES may reduce the number of iterations to lower the computational time [2].

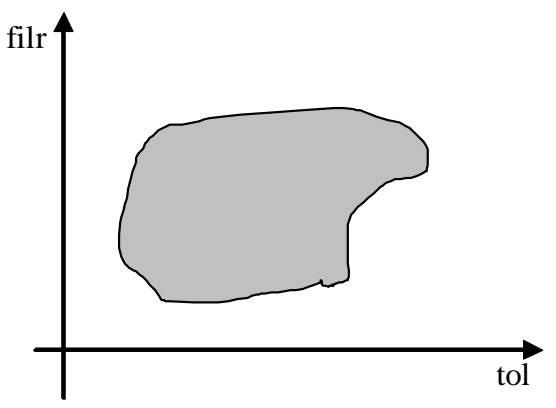

Fig. 2 Parameter space of ILUT.
The drop strategies of ILUT work as follows: (1) Any element in a row whose magnitude is less than tol (relative to the absolute value of the diagonal element of the current row) is dropped. (2) Keeping only the largest lfil elements in the $i$ th row of lower triangular part and the largest lfil element in the $i$ th row of upper triangular part (excluding the diagonal elements) [2]. Corresponding to the two dropping strategies, there are two parameters used in ILUT. One is tolerance value $t o l$, the other is the number of fill-in lfil. Since the lfil parameter may be related to the size of the matrix in question, we use another parameter which is called the fill-in rate filr. Instead of directly assigning a number to lfil, we calculate it by multiplying a given filr value with the ratio of nonzero entries of the row in question. Whether a sparse linear system can be solved by ILUT and the number of iterations the PGMRES will take are closely related to the value of tol and filr. Given a sparse linear system, we want to predict all the possible combinations of tol and filr with which the linear system can be solved. It is a quite hard problem, as both the parameters may take any real positive values, and the possible combination of the parameters may construct arbitrary areas in a two-dimensional parameter space. The left figure in Fig. 2 illustrates the parameter space of ILUT. The closed grey area represents the parameter area within which a certain sparse linear system can be solved. The area may be irregular and/or open and there may exist more than one of such areas. It is very difficult to find some functions to describe such $\operatorname{area}(\mathrm{s})$.

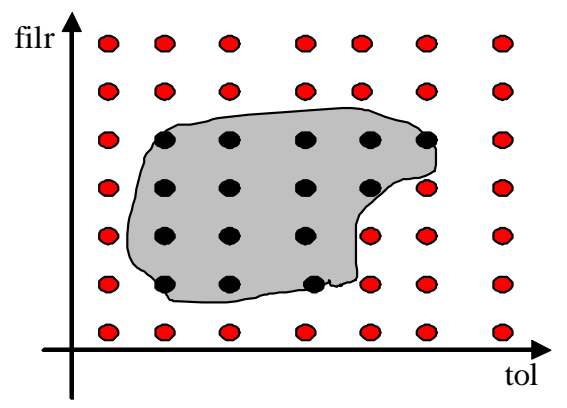


Our idea to solve this problem is to choose some points in the parameter space as samples. Through studying the performance of ILUT with these sample parameters, we can get the main idea of what kinds of combination of the parameters are favorable for a given sparse linear system. In the right figure in Fig. 2, the dots represent samples. If we can correctly predict the solving status at these samples points (red dot means that with the sample parameters the sparse linear system cannot be solved; back dot means it can be solved), we may obtain an outline of the parameter area(s) in which the sparse linear system can be solved. Ideally, if the sample size is large enough and the prediction is accurate, then the shape of the predicted parameter space of solution will approach the real parameter space of solution.

In this paper, we have proposed several methods to predict the solving status at the sample points, which are described below. In many situations the parameter space shows spatial locality, i.e. parameter locations that are closer in parameter space are more likely to have similar solvability. The following methods make usage of spatial locality in different ways.

\subsection{MSC (Multi-points SVM Classifier)}

The first method is a grid based multi-points SVM classifier. SVM is based on structural risk minimization theory [7]. SVM classification has been successfully applied to many applications like face identification, text categorization, bioinformatics, etc. [14].

In this method, we divide the parameter space using a grid and construct SVM classifiers on some grid points (blue squares in Fig. 3). If the sample points on which prediction is to be made are on such grid points, then prediction is made by applying the SVM classifiers directly. If the sample points are not on such grid points (pink diamonds in Fig. 3), then prediction is made by averaging the prediction results on the blue squares within a certain distance. The detailed algorithm is described below.

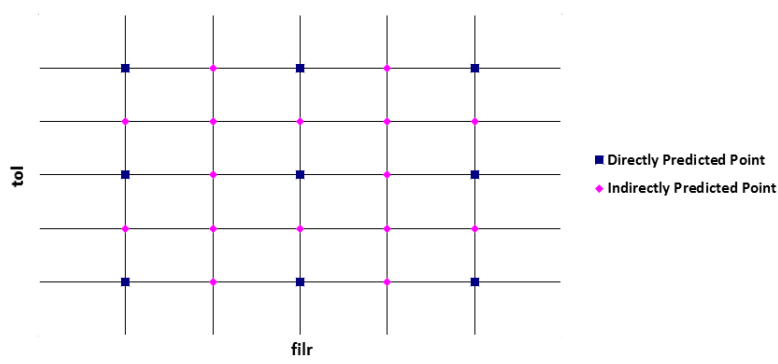

Fig. 3 An illustration of MSC.

Algorithm for MSC:

(1) Choose some sample points in the parameter space;

(2) Build one SVM classifier for each sample point;

(3) If a point on which prediction is to make is the sample point, then make prediction by applying the SVM classifier constructed on the sample point;

(4) If a point $i$ on which prediction is to make is not the sample point, then find all the sample points within distance $\theta$. The prediction result is the weighted sum of the prediction results on the sample points:

$$
p_{i}=\sum_{j=1}^{n} \operatorname{Weight}(\operatorname{Dist}(i, j)) p_{j},
$$

where

$$
\operatorname{Dist}(i, j)<=\theta .
$$

In the formula above, $p_{j}$ is the prediction result on the $j$ th sample point within distance $\theta$. Dist $(i, j)$ is the function to compute the distance between points $i$ and $j$. Weight $(\operatorname{Dist}(i, j))$ is a function to calculate the weight of the prediction on point $j$ based on the distance between $i$ and $j$.

The algorithm for MSC can be used to predict the solving status of ILUT with any given parameter pair. The disadvantages are: it needs to build and train many classifiers. The more the directly predicted points are selected in Fig. 3, the more accurate are the prediction results on the indirectly predicted points. But that means more classifiers need to be built and trained.

\subsection{OSC (Overall SVM Classifier)}

To avoid building and training many classifiers in 
MSC, we propose to use an overall SVM classifier. In this method, sample points are selected as in the previous method. The parameter pair is added to the attributes set and only one classifier is built and trained. To predict the solving status on any point in the parameter space, whether it is on the sample points or not, just input values of the matrix attributes and the parameter pair and then the prediction can be made. The algorithm for this method is listed below:

Algorithm for OSC:

(1) Choose some sample points in the parameter space;

(2) Add the parameter pair to attributes set;

(3) Build and train one overall SVM classifier;

(4) Make prediction with any parameter pair using the SVM classifier.

The advantage of OSC is that it builds and trains only one classifier. Besides, it can directly predict solvability with any parameter pair, saving the efforts in MSC to calculate the distance between points and compute the weighted sum. As the parameter pair is treated as input attributes, the classifier can predict the result with any values the parameters take. The disadvantage is that prediction accuracy is only about $50 \%-70 \%$, which is not satisfactory for many applications.

\subsection{OSAC (Overall Spatial Autoregressive Classifier)}

Spatial autoregressive models [8] are a type of spatial data mining methods. OSAC utilizes spatial autoregressive models which take into consideration the spatial locality property to improve prediction accuracy.

OSAC is similar to OSC in that it builds and trains only one overall classifier. The difference is it applies spatial autoregressive classifier instead of SVM classifier. The algorithm for this method is listed below:

Algorithm for OSAC:

(1) Choose some sample points in the parameter space;

(2) Add the parameter pair to attributes set;
(3) Build one overall classifier using spatial autoregressive model;

(4) Make prediction with any parameter pair using the spatial autoregressive model.

This algorithm can also be used to predict the ILUT solving status with any parameter pair. There is a variety of spatial autoregressive models proposed in literature [15]. Many of the models originate from time series analysis and have been widely applied in econometrics, GIS, etc. We try four of the commonly used models and compare them in the experiments. The four models are described as follows.

\subsubsection{FAR (First-order Spatial AR Model)}

This model is seldom used in real word application, but we choose it because it is the simplest spatial autoregressive model. It only consists of a spatially lagged version of the dependent variable $y[8]$ :

$$
\begin{gathered}
y=\rho W y+\varepsilon, \\
\varepsilon \sim N\left(0, \sigma^{2} I_{n}\right),
\end{gathered}
$$

where the spatial contiguity matrix $W$ has been standardized to have row sums of unity.

\subsubsection{SAR (Spatial Autoregressive Model)}

This model extends the first-order spatial autoregressive model to include a matrix $\mathrm{X}$ of explanatory variables such as those used in traditional regression models. We refer to this model as the SAR following [8]. The SAR model takes the form:

$$
\begin{gathered}
y=\rho W y+X \beta+\varepsilon, \\
\varepsilon \sim N\left(0, \sigma^{2} I_{n}\right),
\end{gathered}
$$

where $y$ contains an $n \times 1$ vector of dependent variables, and $\mathrm{X}$ represents the usual $n \mathrm{x} k$ data matrix containing explanatory variables. The parameter $\rho$ is a coefficient on the spatially lagged dependent variable, $W y$, and the parameter $\beta$ reflects the influence of the explanatory variables on variation in the dependent variable $y$. The model is sometimes termed a mixed regressive spatial autoregressive model because it combines the standard regression model with a spatially lagged dependent variable.

\subsubsection{SEM (Spatial Errors Model)}

In SEM, the basic model is as per the standard 
linear model, but the disturbances exhibit spatial dependence:

$$
\begin{gathered}
y=X \beta+\mu, \\
\mu=\lambda W \mu+\varepsilon, \\
\varepsilon \sim N\left(0, \sigma^{2} I_{n}\right),
\end{gathered}
$$

where the parameter $\lambda$ is a coefficient on the spatially correlated errors analogous to the serial correlation problem in time series models. The parameter $\beta$ reflects the influence of the explanatory variables on variation in the dependent variable $y$. This model is applied when there appears to be significant spatial autocorrelation, but tests for spatial lag effects do not suggest that inclusion of the latter would provide a significant improvement.

\subsubsection{GSM (General Spatial Model)}

The GSM is a general version of the spatial model including both the spatial lagged term as well as a spatially correlated error structure:

$$
\begin{gathered}
y=\rho W_{1} y+X \beta+\mu, \\
\mu=\lambda W_{2} \mu+\varepsilon, \\
\varepsilon \sim N\left(0, \sigma_{\varepsilon}^{2} I_{n}\right),
\end{gathered}
$$

The GSM model looks like a combination of the SAR and the SEM. If there is evidence that spatial dependence exists in the error structure from a SAR model, then GSM model is an appropriate approach to modeling this type of dependence in the errors.

\section{Experiments and Results}

We conduct some experiments to test the prediction methods. The linear systems are constructed by using sparse matrices from MatrixMarket [9]. The right hand sides of the linear systems are constructed by assuming that the solutions are a vector of all ones. The initially guessed solutions are a vector of all zeros. The maximum number of iterations is 500. The convergence stopping criteria is that the 2-norm of the residual vectors is reduced by 7 orders of magnitude. The iterative method used is GMRES(20) and the preconditioner is ILUT. Totally 319 linear systems are tested and the number of attributes is 62 after feature selection. We use SVM Light [17] for SVM classification and RBF kernel is applied. The test results are obtained by using a 4-fold cross validation. To compare the three methods described in Section 3, we adopt the same 25 sample points in the parameter space as in Ref. [6]. For each linear system, about $3 / 4$ of the parameter pairs on the sample points are used for training and building the classifiers, and the other $1 / 4$ of the parameter pairs on the sample points are used for prediction. Then for the next round, another $1 / 4$ of the parameter pairs are chosen for prediction, until finally all the parameter pairs are used once for prediction.

To test and compare the accuracy of the three methods in predicting the parameter solvability space, three metrics are adopted, which are accur, solvrt, and usolvrt. accur measures the overall correct prediction rate, which is calculated as follows:

$$
\text { accur }=\frac{\text { truepositive }+ \text { truenegative }}{\text { truepositive }+ \text { truenegative }+ \text { falsepositve }+ \text { falsenegatve }}
$$

solvrt measures the solvable matrices correct prediction rate, i.e., among the matrices that are predicted to be solvable, the ratio of the matrices that are really solvable.

$$
\text { solvrt }=\frac{\text { truepositive }}{\text { truepositive }+ \text { falsepositve }}
$$

usolvrt measures the unsolvable matrices correct prediction rate, i.e., among the matrices that are predicted to be unsolvable, the ratio of the matrices that are really unsolvable.

$$
\text { usolvrt }=\frac{\text { truenegative }}{\text { truenegative }+ \text { falsenegatve }}
$$

\subsection{Experimental Results for MSC}

To run the algorithm for MSC, we use Chebyshev distance for the $\operatorname{Dist}(i, j)$ function and $\theta$ is set to be 1 . The prediction accuracy for the parameter pairs is shown in Table 1 . The results are obtained by setting 
$\sigma$ to be 0.01 in building the SVM classifiers. The selected sample points are shown in Table 1 as cells with grey background. The accuracy shown on such cells is the average of the accuracy of the 319 matrices predicted directly by applying the SVM classifiers. The accuracy shown on the white cells is calculated by averaging the prediction results of the nearby grey cells with Chebyshev distance no more than 1 .

Table 2 compares the prediction accuracy on the directly predicted cells and indirectly predicted cells. The values in the grey cells are the average prediction accuracy of the 319 matrices on the 9 directly predicted cells. The values in the white cells are the average prediction accuracy on the 16 indirectly predicted cells. It is shown that with all the three different $\sigma$ settings, the indirectly predicted usolvrt is higher than the directly predicted usolvrt, whereas the indirectly predicted solvrt is a little bit lower than the directly predicted solvrt. If the grid shown in Table 1 is used, the indirectly predicted accur is slightly higher than the directly predicted accur.

Fig. 4 shows the influence of $\sigma$, which is used in building the SVM classifiers, on the overall prediction accuracy of MSC. The overall prediction accuracy is the average prediction accuracy of the 319 matrices with 25 different parameter pairs. Compared with usolvrt, solvrt is more affected by the variation of $\sigma$. Accur and solvrt reach the highest value when $\sigma$ is 0.01 , then go down a little bit when $\sigma$ is increased

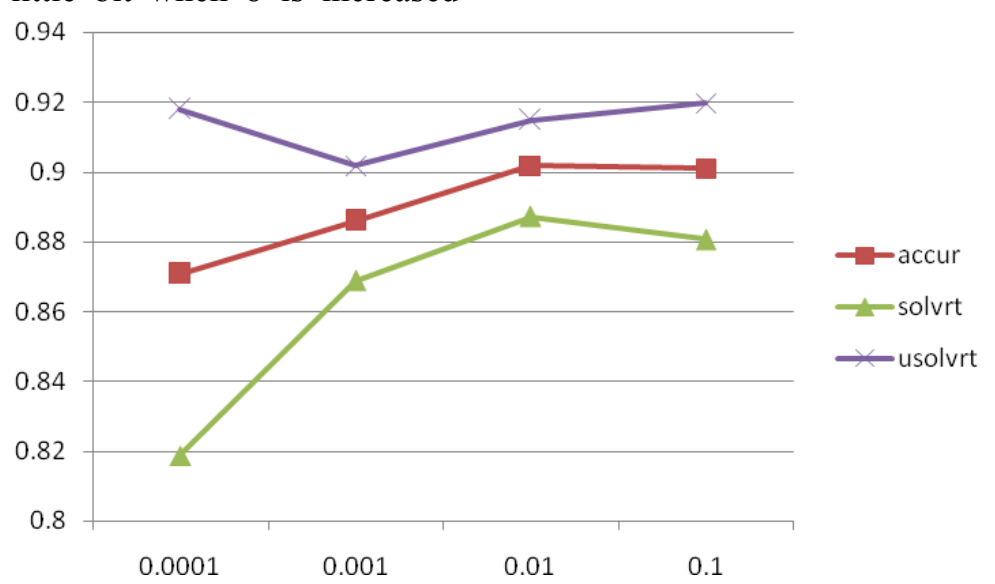

Fig. 4 Prediction accuracy of MSC with different $\sigma$. to 0.1 .

\subsection{Experimental Results for OSC}

The experimental results for OSC are listed in Table 3. The 25 parameter pairs shown in Table 1 are added to the attribute set as input to the SVM classifier. One overall SVM classifier is built and trained and the prediction results are obtained by using a 4-fold cross validation. Table 3 compares the prediction results of OSC with different value of $\sigma$. According to Table 3, the best solvrt is obtained with $\sigma=0.001$, whereas the best usolvrt and accur are obtained with $\sigma=0.1$. The overall prediction accuracy of OSC under the current experimental setting is only about $50 \%$ to $70 \%$.

Table 1 Prediction accuracy of MSC with $\sigma=0.01$.

\begin{tabular}{|c|c|c|c|c|c|}
\hline Tol $=0.1$ & 0.915361 & 0.912226 & 0.918495 & 0.905956 & 0.909091 \\
\hline 0.01 & 0.909091 & 0.905956 & 0.905956 & 0.905956 & 0.899687 \\
\hline 0.001 & 0.905956 & 0.909091 & 0.877743 & 0.887147 & 0.902821 \\
\hline 0.0001 & 0.918495 & 0.893417 & 0.890282 & 0.890282 & 0.893417 \\
\hline 0.00001 & 0.915361 & 0.924765 & 0.893417 & 0.880878 & 0.871473 \\
\hline & filr $=1$ & 2 & 3 & 4 & 5 \\
\hline
\end{tabular}

Table 2 Average prediction accuracy on the directly predicted cells and indirectly predicted cells.

\begin{tabular}{|c|l|l|l|}
\hline Measure & $\sigma=0.001$ & $\sigma=0.01$ & $\sigma=0.1$ \\
\hline \multirow{3}{*}{ accur } & 0.8847 & 0.9011 & 0.9004 \\
\cline { 2 - 4 } & 0.8868 & 0.9020 & 0.9014 \\
\hline \multirow{3}{*}{ solvrt } & 0.8689 & 0.8942 & 0.8888 \\
\cline { 2 - 4 } & 0.8640 & 0.8813 & 0.8748 \\
\hline \multirow{2}{*}{ usolvrt } & 0.8883 & 0.8999 & 0.9048 \\
\cline { 2 - 4 } & 0.9026 & 0.9177 & 0.9218 \\
\hline
\end{tabular}


Table 3 Prediction accuracy of OSC with different $\sigma$.

\begin{tabular}{llll}
\hline Measure & 0.001 & 0.01 & 0.1 \\
\hline accur & 0.5843 & 0.5886 & 0.5999 \\
solvrt & 0.6645 & 0.6256 & 0.4954 \\
usolvrt & 0.5113 & 0.5549 & 0.6951 \\
\hline
\end{tabular}

Table 4 Comparison of the spatial autoregressive models.

\begin{tabular}{lllll}
\hline Measure & FAR & SAR & SEM & GSM \\
\hline accur & 0.5579 & 0.9080 & 0.9083 & 0.8227 \\
solvrt & 0.3826 & 0.8833 & 0.8843 & 0.8207 \\
usolvrt & 0.7176 & 0.9305 & 0.9302 & 0.8245 \\
$R^{2}$ & 0.0123 & 0.6973 & 0.6999 & 0.6996 \\
$\sigma^{2}$ & 0.9856 & 0.2994 & 0.2995 & 0.2998 \\
\hline
\end{tabular}

Table 5 Prediction accuracy of MSC, OSC and OSAC.

\begin{tabular}{llll}
\hline & MSC & OSC & OSAC \\
\hline accur & 0.9017 & 0.5999 & 0.9083 \\
solvrt & 0.8872 & 0.4954 & 0.8843 \\
usolvrt & 0.9149 & 0.6951 & 0.9302 \\
\hline
\end{tabular}

\subsection{Experimental Results for OSAC}

The performance of the OSAC with four different spatial autoregressive models is compared in this section. Besides the measures for accuracy, two more measures are used, which are $R^{2}$ and $\sigma^{2} . R^{2}$ is the coefficient of determination indicating goodness-of-fit of regression. This statistic will be equal to one if fits is perfect, and to zero when regressors $\mathrm{X}$ have no explanatory power. $\sigma^{2}$ is the standard error of regression. The smaller the $\sigma^{2}$ means the better fit of the model.

The comparison of the four spatial autoregressive models is shown in Table 4. The models with the best prediction accuracy are SAR and SEM. The accuracy of the two models is over $90 \%$. Of the matrices that are predicted solvable, $88 \%$ of them are correct; while of the matrices that are predicted unsolvable, $93 \%$ of them are correct. GSM does not fit very well for our experiments with the $82 \%$ accuracy. The prediction accuracy of FAR is the lowest, because it does not take into account the influence of the matrix features at all.

The $R^{2}$ and $\sigma^{2}$ values for the SAR, SEM, and GSM models are similar. The $R^{2}$ values are about 0.7 for these three models which mean the models fit the training data well. However, it does not mean the model can predict the test data well, too. GSM is such an example. But if $R^{2}$ is too low, which means the model cannot even fit the training data well, prediction results is usually poor. FAR is such an example. If $R^{2}$ is low, usually $\sigma^{2}$ is high, which means the standard error of regression is high. For instance, $R^{2}$ is close to zero for FAR, and its $\sigma^{2}$ is close to one.

\subsection{Methods Comparison}

Table 5 compares the prediction accuracy of MSC, OSC, and OSAC. The overall accuracy is obtained by averaging the accuracy of the 319 matrices on the 25 parameter points. The models with the best accur are selected to represent each method. Prediction results with $\sigma=0.01$ are used to represent MSC; prediction results with $\sigma=0.1$ are used to represent OSC; and prediction results with SEM are used to represent OSAC. The prediction accuracy of MSC and OSAC are around $90 \%$. OSAC is slightly better than MSC on accur and usolvrt. The algorithm of OSAC is much simpler to implement compared with MSC. It does not need to build and train many classifiers, find the sample points within distance limit and compute the weighted average to obtain the prediction accuracy. As we mentioned earlier, the accuracy for OSC is only about $50 \%-70 \%$, which motivated us to turn to the spatial autoregressive methods.

\section{Conclusion and Future Work}

We proposed three methods to predict the parameter space of solution for ILUT preconditioned Krylov subspace methods in this paper. The prediction accuracy of MSC and OSAC are both around $90 \%$. OSAC is easier to implement as it builds and trains classifier only once, and it can directly predict solvability with any parameter pair. We focus our work on ILUT preconditioner in this paper, but the proposed strategies should be applicable to other preconditioners with two or more parameters. 
For future works, we plan to modify SVM and OSC to improve prediction accuracy and conduct experiments on other preconditioners with two or more parameters. We also consider trying the deep machine learning algorithms such as convolutional neural network $[10,11]$. To measure prediction accuracy, we will try other metrics such as spatial accuracy (i.e., how far the predictions are from the actual ones) [12] besides the current metrics.

\section{References}

[1] Saad, Y. 1996. Iterative Methods for Space Linear Systems. New York: PWS.

[2] Saad, Y. 1994. "ILUT: A Dual Threshold Incomplete LU Precontioner." Numer. Linear Algebra Appl. 1 (4): 387-402.

[3] Lu, Y., Cohen, I., Zhou, X. S., and Tian, Q. 2007. "Feature Selection Using Principal Feature Analysis." In Proceedings of the 15th International Conference on Multimedia, 301-4.

[4] Zhang, J. 2001. "Performance of ILU Preconditioners for Stationary 3D Navier-Stokes Simulation and the Matrix Mining Project." In Proceedings of the 2001 International Conference on Preconditioning Techniques for Large Sparse Matrix Problems in Scientific and Industrial Applications, Tahoe City, CA, 89-90.

[5] Xu, S., and Zhang, J. 2005. "A Data Mining Approach to Matrix Preconditioning Problem." In Proceedings of Eighth Workshop on Mining Scientific and Engineering Datasets.

[6] Xu, S., and Zhang, J. 2006. "SVM Classification for Predicting Sparse Matrix Solvability with Parameterized Matrix Preconditioners." In Proceedings of the Ninth Workshop on Mining Scientific and Engineering Datasets
(MSD06), in conjunction with the Sixth SIAM International Conference on Data Mining.

[7] Vapnik, V. N. 1998. Statistical Learning Theory. New York: John Wiley and Sons.

[8] LeSage, J. 2009. "The Theory and Practice of Spatial Econometrics." Available at http://www.spatial-econometrics.com, 08/01/2009.

[9] http://math.nist.gov/MatrixMarket.

[10] Van den Oord, A., Dieleman, S., and Schrauwen, B. 2013. "Deep Content-Based Music Recommendation." Curran Associates Inc. 2643-51.

[11] Masakazu, M., Mori, K., Mitari, Y., and Kaneda, Y. 2003. "Subject Independent Facial Expression Recognition with Robust Face Detection Using a Convolutional Neural Network." Neural Networks 16 (5): 555-9.

[12] Shekhar, S., Schrater, P. R., Vatsavai, R. R., Wu, W. L., and Chawla, S. 2002. "Spatial Contextual Classification and Prediction Models for Mining Geospatial Data." IEEE Trans on Multimedia 4 (2): 174-88.

[13] Houstis, E. N., Rice, J. R., and Vichnevetsky, R. 1990. "Intelligent Mathematical Software Systems." In Proceedings of the 1st IMACS/IFAC International Conference on Expert Systems for Numerical Computing, New York.

[14] Joachims, T. 1999. "Making Large-Scale SVM Learning Practical." In Advances in Kernel Methods-Support Vector Learning, edited by Schölkopf, B., Burges, C., and Smola, A. Massachusetts: MIT-Press.

[15] Koperski, K., Adhikary, J., and Han, J. 1996. "Spatial Data Mining: Progress and Challenges-Survey Paper." In Proceedings of SIGMOD Workshop on Research Issues on Data Mining and Knowledge Discovery.

[16] Zhang, J. 2000. "Preconditioned Krylov Subspace Methods for Solving Nonsymmetric Matrices from CFD Applications." Comput. Methods Appl. Mech. Engrg. 189 (3): 825-40.

[17] http://svmlight.joachims.org/. 